\title{
Prognostic significance of the ratio of fibrinogen and albumin in human malignancies: a meta-analysis
}

This article was published in the following Dove Press journal: Cancer Management and Research

\section{Yi Zhang \\ Guoliang Xiao}

Department of General Surgery, The First People's Hospital of Neijiang, Neijiang 641000, Sichuan Province, People's Republic of China

Both authors contributed equally to this work
Correspondence: Yi Zhang

Department of General Surgery, The First People's Hospital of Neijiang, Yuzhong Road, Shizhong District, Neijiang 64I000, Sichuan Province, People's Republic of China

Tel +86I 5282128759

Email 3455647525@qq.com
Background and aim: Serum fibrinogen and albumin are two important factors in systemic inflammation and these two factors are related to tumor progression. This study aimed to comprehensively reveal the prognostic value of the ratio of fibrinogen and albumin in malignant tumors.

Methods: We systematically searched relevant studies in PubMed, Web of Science and Embase up to November 21, 2018. Hazard ratios (HRs) or odds ratio (ORs) for overall survival (OS)/disease-free survival (DFS), as well as relevant clinical data, were collected for analysis; all data analyses were performed by using STATA/SE 14.

Results: Twelve cohort studies were included in this meta-analysis, with a total of 5,088 cases including 9 different kinds of tumors recruited. The pooled results showed that high albumin/fibrinogen ratio (FAR) and low fibrinogen/albumin ratio (AFR) were significantly associated with poor OS (HR=1.50, 95\% CI: 1.30-1.70). Subgroup analyses for OS were also performed based on the disease type, detection method, follow-up time and treatment. Similarly, high FAR or low AFR indicated a worse DFS in cancer patients $(\mathrm{HR}=1.86 ; 95 \%$ CI: 1.41-2.31). In addition, high FAR or low AFR was statistically significant in relation to deeper tumor infiltration $(\mathrm{OR}=2.81,95 \% \mathrm{CI}$ : 1.67-4.72), positive lymph node metastasis $(\mathrm{OR}=1.57,95 \% \mathrm{CI}: 1.23-2.02)$ and distant metastasis $(\mathrm{OR}=2.30,95 \% \mathrm{CI}: 1.36-3.89)$ as well as advanced clinical stage ( $\mathrm{OR}=2.02,95 \% \mathrm{CI}: 1.17-3.47)$.

Conclusions: The ratio of fibrinogen and albumin could act as a promising prognostic marker in human malignant tumors. It might assist physicians to select optimal treatments by identifying the current status of the patient. Future multicenter clinical trials are needed to validate its applications.

Keywords: albumin/fibrinogen ratio, fibrinogen/albumin ratio, malignant tumor, prognosis

\section{Introduction}

Inflammation has been convincingly considered as an important hallmark of cancer. ${ }^{1,2}$ It participates in the initiation, progression, and metastasis of human cancer. ${ }^{3,4}$ Tumorassociated inflammatory factors are closely related to the prognosis in cancer patients. 5,6 Thereof, a series of inflammation-related index systems, including the systemic immune-inflammation index (SII), prognostic nutritional index (PNI) and C-reactive protein/albumin ratio (CAR), were reported as useful predictors in a variety of human tumors. ${ }^{7-9}$ As novel inflammation-based markers, the combination with fibrinogen and albumin, namely, fibrinogen/albumin ratio (FAR) and albumin/fibrinogen ratio (AFR), were proposed and reported currently. The prognostic power of the 
ratio of fibrinogen and albumin was exhibited in several types of human cancers. ${ }^{10-13}$ For example, the patients with high FAR or low AFR had significantly worse survival than those with low FAR or high AFR in the breast, esophageal and gastric cancer; ${ }^{10-12}$ while the ratio of fibrinogen and albumin was found to be no significant impact on overall survival in patients with colorectal cancer. ${ }^{13}$ Besides, the findings on the relationship between the ratio of fibrinogen and albumin and clinicopathologic characteristics were reported but not inconsistent or even contradictory in these studies. Therefore, in order to provide a more accurate understanding of the prognostic significance of circulating FAR or AFR in cancer patients, we performed this systematic review and meta-analysis by pooling all those available data together. The links between the ratio of fibrinogen and albumin and clinicalpathological features were further investigated in this study.

\section{Materials and methods}

\section{Search strategy and study selection}

The current meta-analysis was performed according to the Preferred Reporting Items for Systematic Reviews and Meta-Analyses (PRISMA) guidelines. ${ }^{14}$ PubMed, Embase, and Web of Science were comprehensively searched (up to November 21, 2018) with the following search terms: "albumin-to-fibrinogen or albumin/fibrinogen or fibrinogen-to-albumin or fibrinogen/albumin" combining with "cancer or tumor or malignancy or carcinoma or neoplasms". The search strategy is provided in the Supplementary material.

The inclusion criteria were as follows:

1. The correlation between serum AFR/FAR and overall survival (OS) or disease-free survival (DFS) of primary human malignancies were reported;

2. The assessment of hazard ratios (HRs) and the corresponding 95\% confidence intervals (CIs) was available;

3. The full-articles were published in the English language.

The exclusion criteria were as follows:

1. Non-original articles (reviews, comments, editorials or conference abstracts, or case reports);

2. Articles without sufficient survival data for HRs;

3. Duplicated studies.

\section{Data extraction and quality assessment}

Three aspects of data were collected from the enrolled studies by two authors (Zhang $\mathrm{Y}$ and Xiao GL), independently:

1. Basic information: first author's name, publication year, original country, disease type, the number of enrolled patients, included period, prognostic index, detection method, cut-off value, treatment methods.

2. Clinicopathological features: the distribution of gender, age distribution, histological grade, lymph node metastasis, distant metastasis, tumor invasion depth, clinical stage.

3. Prognostic data: survival type and follow-up time, and due to all included studies reported the HRs for OS/DFS in univariate and/or multivariate analyses, the HRs and 95\% CIs were directly used to combine the individual result and the HRs from multivariate analyses were preferred.

In this study, we focused on two prognostic indicators, AFR and FAR. By definition, these two ratio values are opposite to each other. In this meta-analysis, the HR greater than 1 indicated a worse prognosis in subjects with high FAR or low AFR.

We also assessed the quality of included studies, this method was detailed described in the article by Lin et $\mathrm{al}^{15}$. It contains predefined nine items, a study could get a final score from the lowest 0 and the highest 9 after assessment.

\section{Statistical analysis}

All data analysis was performed using STATA/SE 14.1. The synthesized HRs with $95 \%$ CIs were used to evaluate the prognostic significance of FAR and AFR on OS/DFS in cancer survivors. And the pooled odds ratio (ORs) with 95\% CIs were used to explore the relationship between FAR or AFR and pathological features. Heterogeneity among the enrolled literature was assessed by Higgins ${ }^{2}$ statistics and Cochran's Q. A fixed-effects model was used for non-significant heterogeneity across studies $\left(\mathrm{I}^{2}<50 \%\right.$ and $\left.P>0.1\right)$, otherwise, a random-effects model was utilized. Begg's test, as well as visible plots, were applied to assess the possibility of publication bias. The stability of the overall results was tested by sensitivity analysis, it was conducted by deletion of individual studies one by one. 


\section{Results}

Finally, a total of 12 publications with 5,088 cases were included in this meta-analysis, ${ }^{10-13,16-23}$ the detailed process of study assessment is shown in Figure 1. All these enrolled studies were retrospective design, and they were written in the English language and published in 2017 and 2018. Among them, eleven studies were carried out in P.R. China, and one in Korea. A total of nine different kinds of human malignant tumors were investigated: breast cancer (BC), esophageal squamous cell carcinoma (ESCC), gastric cancer (GC), hepatocellular carcinoma (HCC), non-small cell lung cancer (NSCLC), colorectal cancer (CRC), gallbladder cancer (GBC), chronic lymphocytic leukemia (CLL), and soft tissue sarcoma (STS). Among those studies, seven articles reported the relationship between the AFR and OS, and five studies investigated the association between the FAR and prognosis of OS. Two studies focused on the prognostic value of AFR/FAR on DFS. The quality of all included studies was good with a median score of 8 (range 7-9, Figure 2, Table S1). Table 1 presents the main characteristics of all included studies.
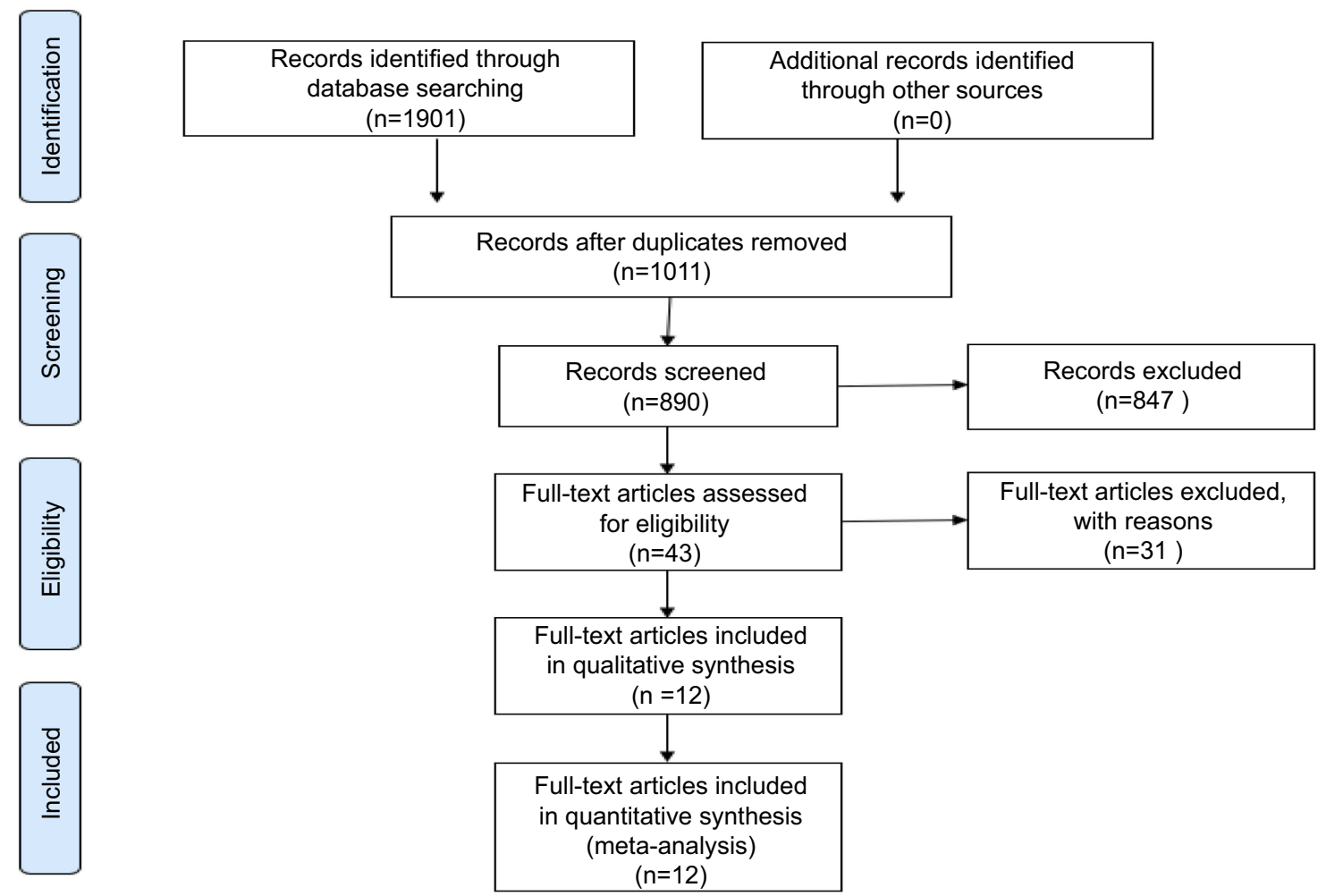

Figure I A flow diagram for the literature assessment process.



Figure 2 Quality assessment of 12 eligible cohort studies.

Abbreviations: OS, overall survival; DFS, disease-free survival; AFR, albumin/fibrinogen ratio; FAR, fibrinogen/albumin ratio. 


\begin{tabular}{|c|c|c|c|c|c|c|c|c|c|c|c|c|c|}
\hline 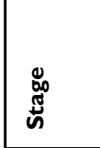 & 景 & $\mathbb{z}$ & $\stackrel{\equiv}{=}$ & 岁 & & $\stackrel{\geq}{\underline{\underline{\underline{I}}}}$ & $\overline{\overline{\underline{\underline{I}}}}$ & $\stackrel{\underline{\underline{I}}}{\underline{\underline{\underline{I}}}}$ & 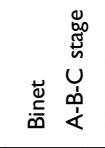 & & $\frac{\overline{\bar{I}}}{\overline{\bar{i}}}$ &  &  \\
\hline  & \multicolumn{6}{|c|}{ 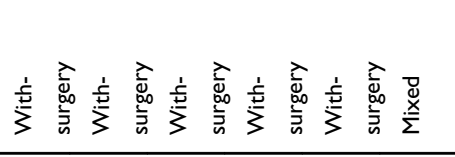 } & \multicolumn{6}{|c|}{ 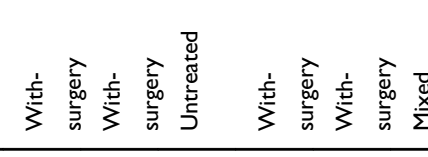 } & \\
\hline \begin{tabular}{|l} 
\\
$\frac{3}{\overline{0}}$ \\
\end{tabular} & 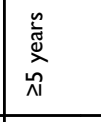 & în & 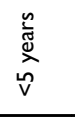 & 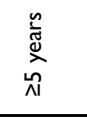 & 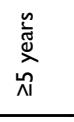 & 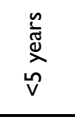 &  & 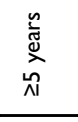 & 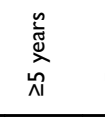 & 点 &  & 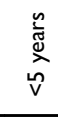 & \\
\hline  & \begin{tabular}{|l}
$\bar{E}$ \\
o
\end{tabular} & 气ू & $\sum_{\tilde{O}}$ & $\sum_{\mathscr{\Sigma}}^{\bar{o}}$ & $\begin{array}{l}\bar{\Sigma} \\
\underline{\underline{\Delta}} \\
\hat{\Sigma} \\
\underline{\Delta}\end{array}$ & $\underset{\Sigma}{\underline{\Delta}}$ & $\underset{\Sigma}{\bar{\varepsilon}}$ & 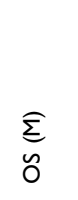 & $\begin{array}{l}\bar{\Sigma} \\
o\end{array}$ & 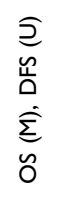 & $\sum_{\mathscr{L}}^{\bar{o}}$ & $\begin{array}{l}\bar{\varepsilon} \\
\varnothing\end{array}$ & o \\
\hline  & $\bar{N}$ & 号 & ${ }_{\infty}^{\infty}$ & 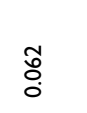 & 官 & $\stackrel{\infty}{\wedge}$ & శٌ & $\stackrel{\infty}{\circ}$ & $\hat{\alpha}$ & : & $m$ & $\stackrel{\text { 导 }}{\underline{\underline{m}}}$ & \\
\hline 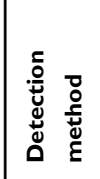 &  &  & 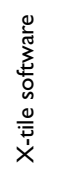 &  &  &  & 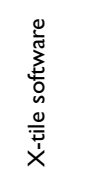 & 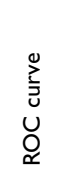 & 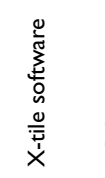 & 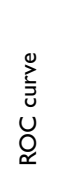 & 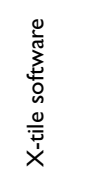 & 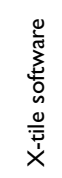 & $\vec{u}$ \\
\hline 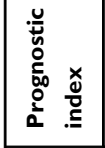 & 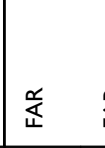 & $\frac{\mathbb{x}}{\underline{x}}$ & 采 & $\underset{\mathbb{\Psi}}{\stackrel{\infty}{\Psi}}$ & 采 & 委 & 委 & $\underset{\mathbb{L}}{\stackrel{\alpha}{\Psi}}$ & 委 & $\stackrel{\alpha}{\Psi}$ & 采 & 采 & \\
\hline 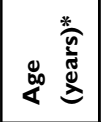 &  & 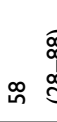 & &  & ๙ & & & & $\overline{0}$ &  & 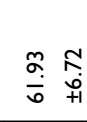 & $\frac{\pi}{z}$ & \\
\hline 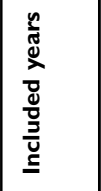 & 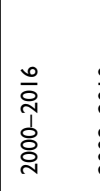 & 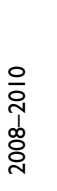 & $\begin{array}{l}\frac{m}{\bar{T}} \\
\overline{\bar{\alpha}}\end{array}$ &  & $\begin{array}{l}\text { 吕 } \\
\bar{T} \\
\text { o }\end{array}$ & 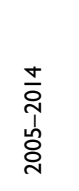 & 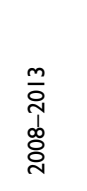 &  & $\begin{array}{l}\hat{\bar{T}} \\
\text { d⿱ } \\
\underline{\alpha}\end{array}$ &  & $\begin{array}{l}\frac{m}{\bar{a}} \\
\frac{1}{\bar{i}}\end{array}$ &  & 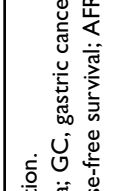 \\
\hline  & 辤 & 鹳 & $\frac{\alpha}{\bar{g}}$ & $\underset{\widetilde{\Phi}}{\stackrel{\widetilde{\Phi}}{\simeq}}$ & $\stackrel{\overline{\hat{I}}}{\frac{N}{m}}$ & $\frac{\alpha}{\frac{\alpha}{m}}$ & 壱 & 产 & 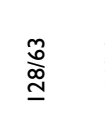 & 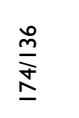 & 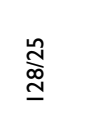 & $\stackrel{\infty}{\text { ป }}$ & \\
\hline  & $\stackrel{m}{R}$ & $\stackrel{\stackrel{m}{m}}{=}$ & $\stackrel{\circ}{\circ}$ & $\underline{\underline{n}}$ & సิ సి & $\stackrel{\sim}{F}$ & జ్ర & 吉 & $\bar{\varrho}$ & $\frac{\circ}{m}$ & $\underline{\underline{n}}$ & స్తి & \\
\hline 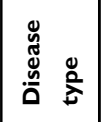 & 品 & 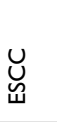 & u & $\underset{\underline{Y}}{\underline{I}}$ & 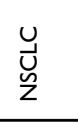 & $\begin{array}{l}\breve{u} \\
\underline{u} \\
\end{array}$ & Ü & Ü & $\vec{u}$ & $\stackrel{n}{5}$ & 崫 & 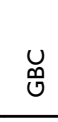 & \\
\hline 譬 & 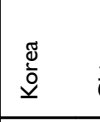 & 兽 & 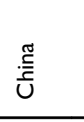 & $\frac{\tilde{c}}{\tilde{z}}$ & 絯 & 苞 & $\frac{\tilde{g}}{\tilde{\bar{E}}}$ & 趇 & 䗆 & 尊 & 㨱 & 疍 & \\
\hline : & $\overline{\bar{a}}$ & 率 & ⿳े & $\stackrel{\infty}{\grave{\alpha}}$ & $\frac{\infty}{a}$ & $\stackrel{\infty}{a}$ & $\stackrel{\infty}{\grave{c}}$ & $\stackrel{\infty}{\grave{c}}$ & $\stackrel{\infty}{i}$ & $\frac{\infty}{2}$ & $\stackrel{\infty}{\grave{\alpha}}$ & $\stackrel{\infty}{i}$ & \\
\hline 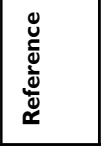 & 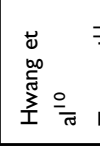 & & 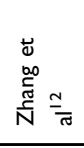 & $\begin{array}{l}\frac{0}{\bar{J}} \\
\stackrel{0}{\Delta} \\
\vec{x} \\
\end{array}$ & 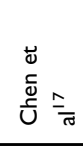 &  &  & $\begin{array}{l}\frac{\alpha}{\bar{\sigma}} \\
\tilde{J} \\
\overrightarrow{\bar{x}} \\
\end{array}$ & $\begin{array}{l}\frac{2}{\bar{d}} \\
\overline{0} \\
\overline{0} \\
\\
\end{array}$ & 齿 $\frac{\bar{\alpha}}{\sigma}$ & 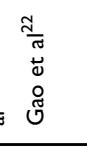 & $\begin{array}{l}\frac{\pi}{\sigma} \\
\bar{\sigma} \\
\bar{\Xi} \\
\end{array}$ & \\
\hline
\end{tabular}




\section{The ratio of fibrinogen and albumin and OS}

12 studies involving 5088 cases described the association between the ratio of fibrinogen and albumin and OS. No significant heterogeneity existed across studies $\left(\mathrm{I}^{2}=30.7 \%\right.$, $P=0.145$ ), the fixed effects model was applied to merge the HRs and 95\% CIs. The meta-analysis results showed that high FAR or low AFR was obviously correlated with poor survival in cancer patients $(\mathrm{HR}=1.50,95 \% \mathrm{CI}: 1.30-1.70 ; P<0.001$, Figure 3).

Furthermore, subgroup analyses were conducted based on the disease type, detection method, follow-up time and treatment. We found that high FAR or low AFR was closely correlated with shorter OS in gastrointestinal (GI) cancers $(\mathrm{HR}=1.40 ; 95 \% \mathrm{CI}: 1.17-1.62 ; P<0.001)$ and non-GI cancers $(\mathrm{HR}=1.96 ; 95 \% \mathrm{CI}: 1.49-2.42 ; P<0.001)$. For the

\begin{tabular}{|c|c|c|c|}
\hline \multicolumn{2}{|l|}{$\begin{array}{l}\text { Study } \\
\text { ID }\end{array}$} & $\mathrm{HR}(95 \% \mathrm{Cl})$ & $\begin{array}{l}\% \\
\text { Weight }\end{array}$ \\
\hline Hwang $\mathrm{KT}^{10}$ & $\longrightarrow$ & $2.62(1.46,4.72)$ & 1.52 \\
\hline $\operatorname{Tan} Z^{11}$ & $\leftarrow$ & $1.33(1.09,1.69)$ & 44.52 \\
\hline Zhang $\mathrm{J}^{12}$ & $\rightarrow-$ & $1.54(1.01,2.34)$ & 9.18 \\
\hline Xu QD 16 & $i$ & $2.02(1.27,3.21)$ & 4.31 \\
\hline Chen $\mathrm{S}^{17}$ &  & $1.95(1.21,3.16)$ & 4.24 \\
\hline $\mathrm{Li} \mathrm{SQ}^{18}$ & $\rightarrow$ & $1.79(1.23,2.61)$ & 8.53 \\
\hline Sun $\mathrm{F}^{13}$ & $\because-$ & $1.41(0.90,2.20)$ & 9.70 \\
\hline $\mathrm{Xu} W \mathrm{Y}^{19}$ & $\longrightarrow$ & $2.81(1.76,4.48)$ & 2.20 \\
\hline Zou $Y X^{20}$ & & $3.47(1.54,7.82)$ & 0.41 \\
\hline Liang $Y X^{21}$ & 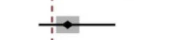 & $1.91(1.16,3.13)$ & 4.18 \\
\hline Gao QF 22 & 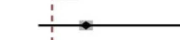 & $2.38(1.15,4.93)$ & 1.14 \\
\hline $\mathrm{Du} \mathrm{JH}^{23}$ & $\rightarrow$ & $0.89(0.46,1.73)$ & 10.07 \\
\hline Overall (I-squared $=30.70 \%, P=0.145)$ & 0 & $1.50(1.30,1.70)$ & 100.00 \\
\hline-7.82 & & & \\
\hline
\end{tabular}

Figure 3 Pooled results for the correlation between the ratio of fibrinogen and albumin and OS in patients suffering from malignant tumors. Abbreviations: OS, overall survival; $\mathrm{HR}$, hazard ratio; $\mathrm{Cl}$, confidence interval.

Table 2 Subgroup analysis for OS in the patient with malignant tumors

\begin{tabular}{|c|c|c|c|c|c|c|}
\hline \multirow[t]{2}{*}{ Stratified analysis } & \multirow[t]{2}{*}{ Studies (n) } & \multicolumn{3}{|c|}{ Pooled results } & \multicolumn{2}{|c|}{ Heterogeneity } \\
\hline & & HR & $95 \% \mathrm{Cl}$ & $P$-value & $I^{2}(\%)$ & $P$ \\
\hline \multicolumn{7}{|l|}{ Tumor type } \\
\hline GI cancer & 7 & 1.40 & $1.17-1.62$ & $<0.001$ & 37.6 & 0.142 \\
\hline Non-Gl cancer & 5 & 1.96 & $1.49-2.42$ & $<0.001$ & 0.0 & 0.780 \\
\hline \multicolumn{7}{|l|}{ Detection method } \\
\hline ROC curve & 5 & 2.13 & $1.64-2.63$ & $<0.001$ & 0.0 & 0.791 \\
\hline X-tile software & 7 & 1.38 & $1.16-1.60$ & $<0.001$ & 11.4 & 0.342 \\
\hline \multicolumn{7}{|l|}{ Follow-up time } \\
\hline$<5$ years & 5 & 1.42 & $1.09-1.74$ & $<0.001$ & 18.4 & 0.297 \\
\hline$\geq 5$ years & 7 & 1.56 & $1.30-1.81$ & $<0.001$ & 43.1 & 0.103 \\
\hline \multicolumn{7}{|l|}{ Treatment situation } \\
\hline With-surgery & 9 & 1.54 & $1.31-1.76$ & $<0.001$ & 20.3 & 0.262 \\
\hline Mixed & 2 & 1.30 & $0.84-1.77$ & NS & 71.7 & 0.060 \\
\hline Untreated & 1 & 3.47 & $1.54-7.82$ & $<0.001$ & - & - \\
\hline
\end{tabular}

Abbreviations: OS, overall survival; HR, hazard ratio; $\mathrm{Cl}$, confidence interval; Gl, gastrointestinal; ROC, receiver operating characteristic, NS, not significant. 
subgroup involving detection methods, it was observed that patients with high FAR or low AFR had a poorer OS in both groups using ROC curve (HR=2.13; 95\% CI: 1.64-2.63; $P<0.001)$ and $\mathrm{X}$-tile software $(\mathrm{HR}=1.38 ; 95 \% \mathrm{CI}$ : $1.16-1.60 ; P<0.001)$. Similarly, high FAR and low AFR were significantly related to shorter OS in group with followups $\geq 5$ years $(\mathrm{HR}=1.56 ; 95 \% \mathrm{CI}: 1.30-1.81 ; P<0.001)$ and follow-ups $<5$ years $(\mathrm{HR}=1.42 ; 95 \%$ CI: 1.09-1.74; $P<0.001)$. Obviously, high FAR and low AFR could serve as significant unfavorable factors in patients after curative resection $(\mathrm{HR}=1.54 ; 95 \% \mathrm{CI}: 1.31-1.76 ; P<0.001)$. All results of subgroup analyses are presented in Table 2.

\section{The ratio of fibrinogen and albumin and DFS}

Only two studies reported the relationship between the ratio of fibrinogen and albumin and DFS, no significant heterogeneity was found among studies $\left(\mathrm{I}^{2}=0.0 \%\right.$,
$P=0.707$ ), a fixed effects model was used to analyze the pooled HRs. As shown in Figure 4, the combined results indicated that the patients with high FAR or low AFR had a worse DFS (HR=1.86; 95\% CI: $1.41-2.31 ; P<0.001$ ).

\section{The ratio of fibrinogen and albumin and clinicopathological features}

The clinical relevance of the ratio of fibrinogen and albumin in tumor cases is presented in Table 3. High FAR and low AFR were found to be significantly correlated with deeper infiltration (OR=2.81, 95\%CI: $1.67-4.72, P=0.000)$, positive lymph node metastasis (OR=1.57, 95\%CI: 1.23-2.02, $P=0.000)$, distant metastasis $(\mathrm{OR}=2.30,95 \%$ CI: $1.36-3.89, P=0.002)$ and advanced clinical stage (OR=2.02, 95\%CI: $1.17-3.47, P=0.011)$, whereas the high FAR or low AFR was not statistically significantly associated with histological grade $(\mathrm{OR}=1.29,95 \% \mathrm{CI}$ : $0.94-1.76, P=0.115)$.

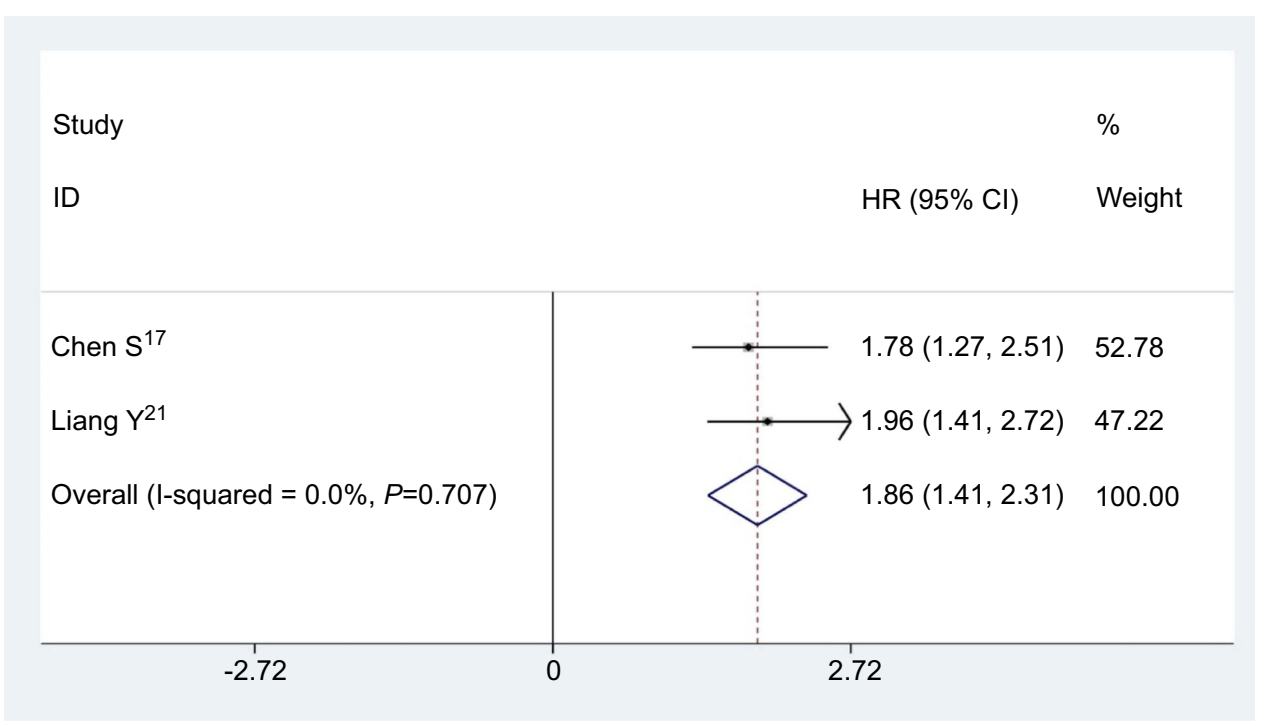

Figure 4 Pooled results for the correlation between the ratio of fibrinogen and albumin and DFS in cancer patients.

Abbreviations: DFS, disease-free survival; $\mathrm{HR}$, hazard ratio; $\mathrm{Cl}$, confidence interval.

Table 3 Results of the meta-analysis of clinicopathological characteristics

\begin{tabular}{|c|c|c|c|c|c|c|c|}
\hline \multirow{2}{*}{$\begin{array}{l}\text { Clinicopathological } \\
\text { parameter }\end{array}$} & \multirow[t]{2}{*}{ Studies (n) } & \multicolumn{3}{|c|}{ Pooled results } & \multicolumn{3}{|c|}{ Heterogeneity } \\
\hline & & OR & $95 \% \mathrm{Cl}$ & $P$-value & $I^{2}(\%)$ & $\boldsymbol{P}$ & Model \\
\hline Histological grade (G3/G4 vs.GI/G2) & 8 & 1.29 & $0.94-1.76$ & 0.115 & 67.0 & 0.004 & Random \\
\hline Infiltration (T3-4 vs Tis-I-2) & 6 & 2.81 & $1.67-4.72$ & $<0.001$ & 73.6 & 0.002 & Random \\
\hline Lymph node metastasis (+ vs -) & 7 & 1.57 & $1.23-2.02$ & $<0.001$ & 55.7 & 0.035 & Random \\
\hline Distant metastasis (+ vs -) & 2 & 2.30 & $1.36-3.89$ & 0.002 & 0.0 & 0.771 & Fixed \\
\hline Clinical stage (III-IV vs 0-I-II) & 6 & 2.02 & I. $17-3.47$ & 0.011 & 77.3 & 0.001 & Random \\
\hline
\end{tabular}

Abbreviations: OR, odds ratio; $\mathrm{Cl}$, confidence interval. 


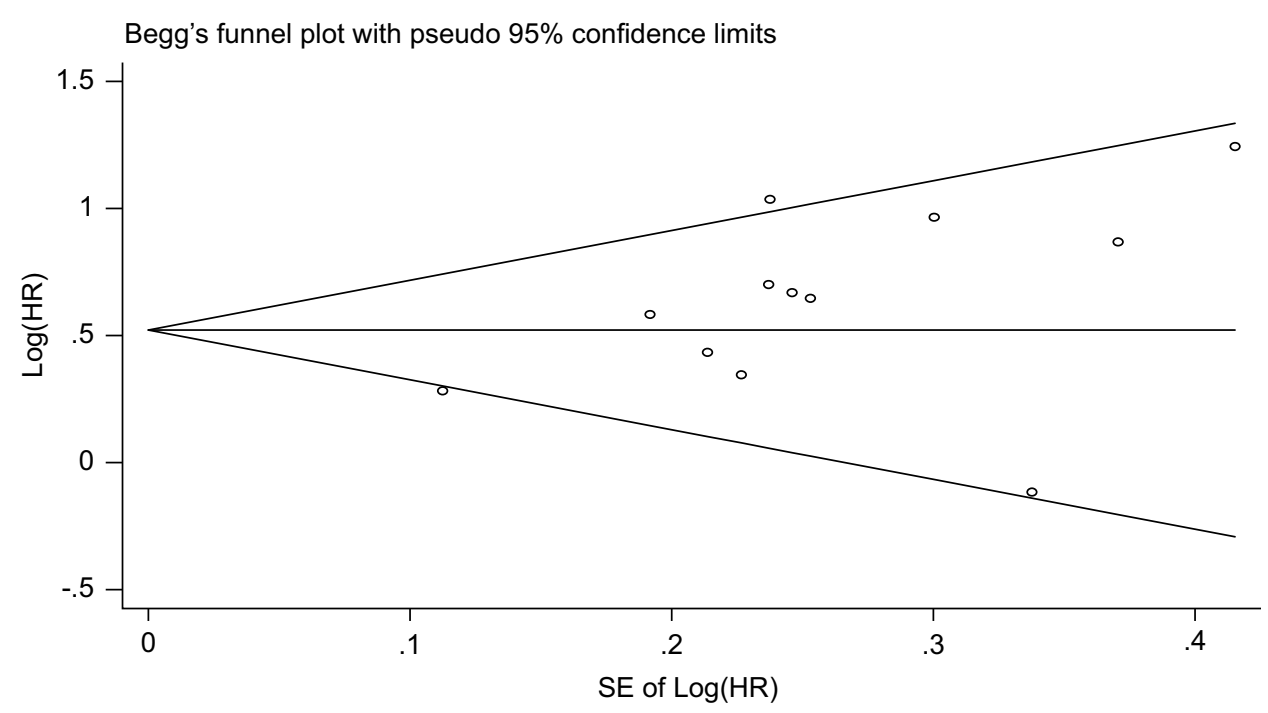

Figure 5 Begg's funnel plot for OS.

Abbreviations: OS, overall survival; HR, hazard ratio.

\section{Publication bias}

Begg's funnel plot was symmetrical (Figure 5) and Begg's test had a value of $0.086(P>0.05)$, which suggestive of the absence of publication bias.

\section{Sensitivity analysis}

Sensitivity analysis indicated that the overall results in this meta-analysis were stable and reliable (Figure 6).

\section{Discussion}

The relationships between FAR and AFR and prognosis were reported in previous studies, however, their prognostic and clinical-pathological values in cancer patients remains undetermined. Hence, we performed this metaanalysis for the first time to figure out the prognostic impact of this scoring system in cancers. We included 12 cohort studies with a total of 5088 cancer cases, and 9 different kinds of human malignancies were enrolled. By

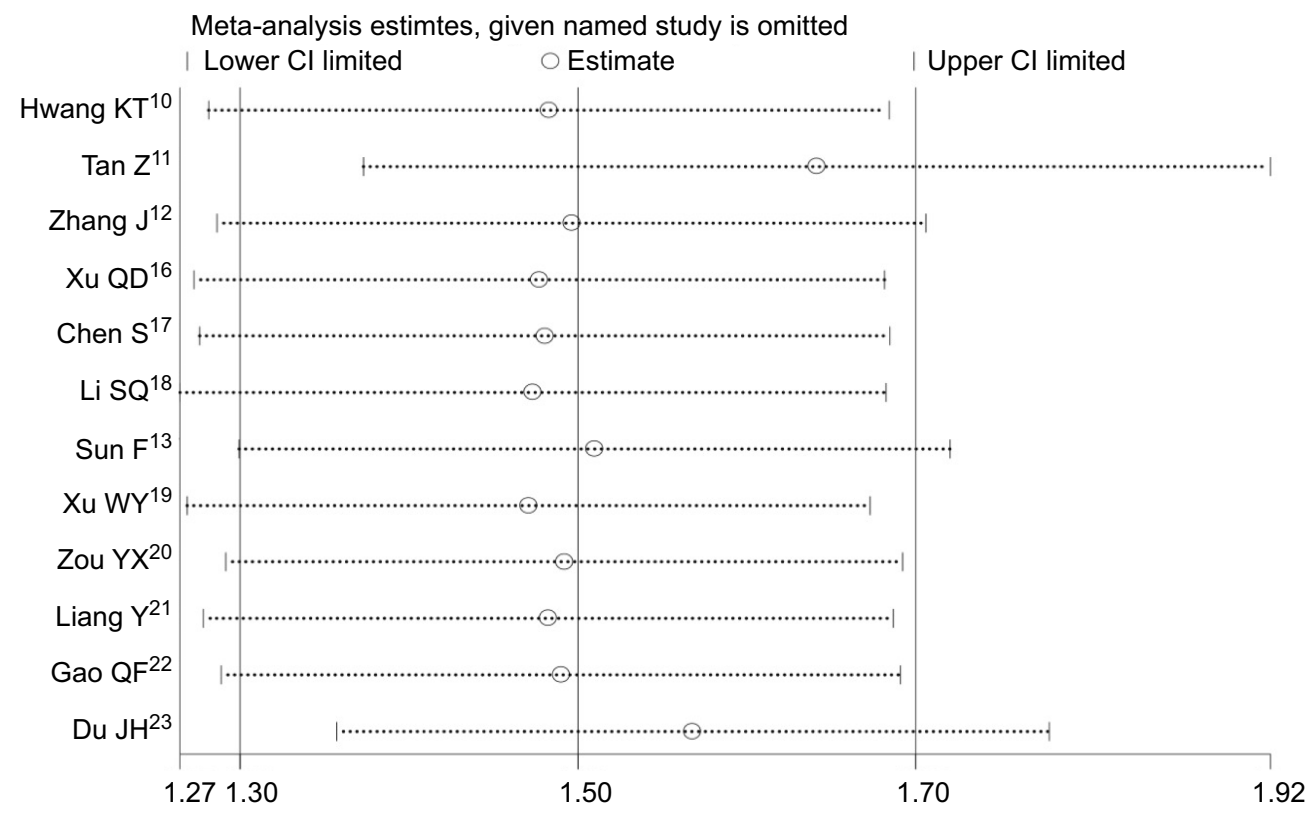

Figure 6 Sensitivity analysis test for OS.

Abbreviations: $\mathrm{OS}$, overall survival; $\mathrm{Cl}$, confidence interval. 
pooling all the available OS data from multivariate analyses, we found that patients with high FAR or low AFR had a significantly lower OS rate than those with low FAR or high AFR (HR=1.50, 95\% CI: 1.30-1.70; $p<0.001$ ), and moreover, high FAR or low AFR was found to be an independent factor of unfavorable OS in patients with GI cancers or non-GI cancers, and there was clear evidence that high FAR and low AFR could be a significant predictor in long-term overall survival rate ( $\geq 5$ years) or short-term survival rate $(<5$ years) between the two groups. In addition, the ratio of fibrinogen and albumin might be an important predictor for DFS in cancers $\quad(\mathrm{HR}=1.86 ; \quad 95 \%$ CI: $1.41-2.31 ; p<0.001)$. Similarly, the significant associations between clinicopathological features and this scoring system were also found in tumor infiltration $(p<0.001)$, lymph node metastasis $(p<0.001)$, distant metastasis $(p=0.002)$ and clinical stage $(p=0.011)$.

Although the specific mechanism for the roles of this scoring system predicting cancer progression and prognosis remains incompletely understood, there are some potential explanations for this. Fibrinogen level was frequently observed elevated in serum of the cancer patients and hyper-fibrinogenemia was demonstrated to be related to unfavorable prognoses in multiple tumors, including liver cancer, lung cancer, esophageal cancer and urinary tract urothelial carcinoma. ${ }^{24-27}$ Fibrinogen is an important factor involved in hemostasis, and the coagulation system is often abnormally activated in cancer patients, ${ }^{2,28,29}$ and it could possess anti-cancer properties as a compound with sodium selenite. ${ }^{6}$ And as an acute-phase-response protein, fibrinogen could also reflect the statue of the systemic inflammatory response, which was closely related to carcinogenesis. ${ }^{30-32}$ In addition, fibrinogen could regulate tumor cell growth as an extracellular matrix protein by binding to multiple growth factors, ${ }^{33-35}$ and enhance cell migration, invasion, and metastasis involving epithelialmesenchymal transition. ${ }^{36-38}$ Fibrinogen also played a vital role in angiogenesis, which closely participated in the progression of cancer. ${ }^{39-42}$

On the other side, albumin has been considered as an indicator that could objectively reflect the nutritional status, low serum albumin level often heralds malnutrition, and indicates weakened immunity in subjects. ${ }^{43-45}$ In addition, albumin is also a crucial factor that involved in the systemic inflammatory response, low concentration of albumin was shown to be related to the enhancement of inflammatory response to cancers and then release of a series of cancer-related cytokines, which could contribute to tumor development. ${ }^{46-48}$ Furthermore, clinical studies showed that there was a significant association between albumin level and prognosis in various types of tumors, the low pretreatment serum albumin level yielded an unfavorable prognosis in cancers. ${ }^{49-51}$

Several limitations in our meta-analysis should be carefully considered. First, the number of cohort studies that included was relatively small and the total sample size was also limited. Second, all included studies were carried out in two Asian countries, this might affect the applicability of our conclusions, more studies with different populations are required in the future. Third, we only included full-text articles published in the English language. Fourth, the prognostic roles of the ratio of fibrinogen and albumin in specific cancers were not investigated for the lack of sufficient data. Fifth, only two studies reported the data of DFS rate, and as a result, their impacts on DFS should be further confirmed. Last but not least, the cut-off of this scoring system varied among studies. As the limitations mentioned above, further researches should be embraced to verify and update our findings.

In summary, this meta-analysis systematically assessed the prognostic impact of the novel inflammation-based prognostic scores (FAR and AFR) on cancer patients. We found that high FAR and low AFR indicated worse prognosis and adverse clinical progression in tumors. And the prognostic validity of AFR and FAR should be globally checked for various cancer types, as these parameters can be easily obtained in a cheap and non-invasive manner, this might lead to their incorporation in official cancer management guidelines.

\section{Disclosure}

The authors report no conflicts of interest in this work.

\section{References}

1. Mantovani A, Allavena P, Sica A, Balkwill F. Cancer-related inflammation. Nature. 2008;454:436-444. doi:10.1038/nature07205

2. Grivennikov SI, Greten FR, Karin M. Immunity, inflammation, and cancer. Cell. 2010;140:883-899. doi:10.1016/j.cell.2010.01.025

3. Balkwill F, Mantovani A. Inflammation and cancer: back to Virchow? Lancet. 2001;357:539-545. doi:10.1016/S0140-6736(00)04046-0

4. Diakos CI, Charles KA, McMillan DC, Clarke SJ. Cancer-related inflammation and treatment effectiveness. Lancet Oncol. 2014;15: e493-e503. doi:10.1016/S1470-2045(14)70263-3

5. Colotta F, Allavena P, Sica A, Garlanda C, Mantovani A. Cancerrelated inflammation, the seventh hallmark of cancer: links to genetic instability. Carcinogenesis. 2009;30:1073-1081. doi:10.1093/carcin/ bgp 127 
6. Kieliszek M, Lipinski B, Błażejak S. Application of sodium selenite in the prevention and treatment of cancers. Cells. 2017;6:E39. doi:10.3390/cells6040039

7. Zhang Y, Lin S, Yang X, Wang R, Luo L. Prognostic value of pretreatment systemic immune-inflammation index in patients with gastrointestinal cancers. $J$ Cell Physiol. 2019;234:5555-5563. doi:10.1002/jcp. 27373

8. Sun K, Chen S, Xu J, Li G, He Y. The prognostic significance of the prognostic nutritional index in cancer: a systematic review and meta-analysis. J Cancer Res Clin Oncol. 2014;140:1537-1549. doi:10.1007/s00432-014-1714-3

9. Li N, Tian GW, Wang Y, Zhang H, Wang Z-H, Li G. Prognostic role of the pretreatment $\mathrm{C}$-reactive protein/albumin ratio in solid cancers: a meta-analysis. Sci Rep. 2017;7:41298. doi:10.1038/srep41298

10. Hwang KT, Chung JK, Roh EY, et al. Prognostic influence of preoperative fibrinogen to albumin ratio for breast cancer. $J$ Breast Cancer. 2017;20:254-263. doi:10.4048/jbc.2017.20.3.254

11. Tan Z, Zhang M, Han Q, et al. A novel blood tool of cancer prognosis in esophageal squamous cell carcinoma: the fibrinogen/albumin ratio. $J$ Cancer. 2017;8:1025-1029. doi:10.7150/jca.16491

12. Zhang J, Li SQ, Liao ZH, et al. Prognostic value of a novel FPR biomarker in patients with surgical stage II and III gastric cancer Oncotarget. 2017;8:75195-75205. doi:10.18632/oncotarget.20661

13. Sun F, Peng HX, Gao QF, et al. Preoperative circulating FPR and CCF score are promising biomarkers for predicting clinical outcome of stage II-III colorectal cancer patients. Cancer Manag Res. 2018;10:2151-2161. doi:10.2147/CMAR.S167398

14. Moher D, Liberati A, Tetzlaff J, Altman DG. Preferred reporting items for systematic reviews and meta-analyses: the PRISMA statement. PLoS Med. 2009;6:e1000097. doi:10.1371/journal.pmed.1000097

15. Lin Y, Liu Z, Qiu Y, et al. Clinical significance of plasma D-dimer and fibrinogen in digestive cancer: a systematic review and meta-analysis. Eur J Surg Oncol. 2018;44:1494-1503. doi:10.1016/ j.ejso.2018.07.052

16. Xu Q, Yan Y, Gu S, et al. A novel inflammation-based prognostic score: the fibrinogen/albumin ratio predicts prognoses of patients after curative resection for hepatocellular carcinoma. J Immunol Res. 2018;2018:1-11. doi:10.1155/2018/4925498

17. Chen S, Yan H, Du J, et al. Prognostic significance of pre-resection albumin/fibrinogen ratio in patients with non-small cell lung cancer: a propensity score matching analysis. Clin Chim Acta. 2018;482:203-208. doi:10.1016/j.cca.2018.04.012

18. Li SQ, Jiang YH, Lin J, et al. Albumin-to-fibrinogen ratio as a promising biomarker to predict clinical outcome of non-small cell lung cancer individuals. Cancer Med. 2018;7:1221-1231. doi:10.1002/cam4.1428

19. Xu WY, Zhang HH, Xiong JP, et al. Prognostic significance of the fibrinogen-to-albumin ratio in gallbladder cancer patients. World $J$ Gastroenterol. 2018;24:3281-3292. doi:10.3748/wjg.v24.i29.3281

20. Zou YX, Qiao J, Zhu HY, et al. Albumin-to-fibrinogen ratio as an independent prognostic parameter in untreated chronic lymphocytic leukemia: a retrospective study of 191 cases. Cancer Res Treat. 2018. doi:10.4143/crt.2018.358

21. Liang Y, Wang W, Que Y, et al. Prognostic value of the fibrinogen/ albumin ratio (FAR) in patients with operable soft tissue sarcoma. BMC Cancer. 2018;18:942. doi:10.1186/s12885-018-4242-8

22. Gao QF, Qiu JC, Huang XH, et al. The predictive and prognostic role of a novel ADS score in esophageal squamous cell carcinoma patients undergoing esophagectomy. Cancer Cell Int. 2018;18:153. doi:10.1186/s12935-018-0648-2

23. Du JH, Lu J. Circulating CEA-dNLR score predicts clinical outcome of metastatic gallbladder cancer patient. J Clin Lab Anal. 2019;33: e22684. doi:10.1002/jcla.22658

24. Huang G, Jiang H, Lin Y, et al. Prognostic value of plasma fibrinogen in hepatocellular carcinoma: a meta-analysis. Cancer Manag Res. 2018;10:5027-5041. doi:10.2147/CMAR.S175780
25. Zhong H, Qian Y, Fang S, Wang Y, Tang Y, Gu W. Prognostic value of plasma fibrinogen in lung cancer patients: a meta-analysis. $J$ Cancer. 2018;9:3904-3911. doi:10.7150/jca.26360

26. Wang J, Liu H, Shao N, et al. The clinical significance of preoperative plasma fibrinogen level and platelet count in resectable esophageal squamous cell carcinoma. World J Surg Oncol. 2015;13:157. doi:10.1186/s12957-015-0543-4

27. Pichler M, Dalpiaz O, Ehrlich GC, et al. Validation of the preoperative plasma fibrinogen level as a prognostic factor in a European cohort of patients with localized upper tract urothelial carcinoma. $J$ Urol. 2014;191:920-925. doi:10.1016/j. juro.2013.10.073

28. Kieliszek M, Lipinski B. Pathophysiological significance of protein hydrophobic interactions: an emerging hypothesis. Med Hypotheses. 2018;110:15-22. doi:10.1016/j.mehy.2017.10.021

29. McMillan DC. The systemic inflammation-based Glasgow Prognostic Score: a decade of experience in patients with cancer. Cancer Treat Rev. 2013;39:534-540. doi:10.1016/j.ctrv.2012.08.003

30. Qi Q, Geng Y, Sun M, Chen H, Wang P, Chen Z. Hyperfibrinogen is associated with the systemic inflammatory response and predicts poor prognosis in advanced pancreatic cancer. Pancreas. 2015;44:977-982. doi:10.1097/MPA.0000000000000353

31. Dolan RD, McSorley ST, Horgan PG, Laird B, McMillan DC. The role of the systemic inflammatory response in predicting outcomes in patients with advanced inoperable cancer: systematic review and meta-analysis. Crit Rev Oncol Hematol. 2017;116:134-146. doi:10.1016/j.critrevonc.2017.06.002

32. Lipinski B. Rationale for the treatment of cancer with sodium selenite. Med Hypotheses. 2005;64:806-810. doi:10.1016/j. mehy.2004.10.012

33. Sahni A, Francis CW. Vascular endothelial growth factor binds to fibrinogen and fibrin and stimulates endothelial cell proliferation. Blood. 2000;96:3772-3778.

34. Witsch E, Sela M, Yarden Y. Roles for growth factors in cancer progression. Physiology. 2010;25:85-101. doi:10.1152/ physiol.00045.2009

35. Adams GN, Rosenfeldt L, Frederick M, et al. Colon cancer growth and dissemination relies upon thrombin, stromal PAR-1, and fibrinogen. Cancer Res. 2015;75:4235-4243. doi:10.1158/00085472.CAN-15-0964

36. Zhang F, Wang Y, Sun P, et al. Fibrinogen promotes malignant biological tumor behavior involving epithelial-mesenchymal transition via the p-AKT/p-mTOR pathway in esophageal squamous cell carcinoma. J Cancer Res Clin Oncol. 2017;143:2413-2424. doi:10.1007/s00432-017-2493-4

37. Steinbrecher KA, Horowitz NA, Blevins EA, et al. Colitis-associated cancer is dependent on the interplay between the hemostatic and inflammatory systems and supported by integrin alpha(M)beta(2) engagement of fibrinogen. Cancer Res. 2010;70:2634-2643. doi:10.1158/0008-5472.CAN-09-3465

38. Shu YJ, Weng H, Bao RF, et al. Clinical and prognostic significance of preoperative plasma hyperfibrinogenemia in gallbladder cancer patients following surgical resection: a retrospective and in vitro study. BMC Cancer. 2014;14:566. doi:10.1186/1471-240714-566

39. Dvorak HF, Harvey VS, Estrella P, Brown LF, McDonagh J, Dvorak AM. Fibrin containing gels induce angiogenesis. Implications for tumor stroma generation and wound healing. Lab Invest. 1987;57:673-686.

40. Zhao C, Su Y, Zhang J, et al. Fibrinogen-derived fibrinostatin inhibits tumor growth through anti-angiogenesis. Cancer Sci. 2015;106:1596-1606. doi:10.1111/cas.12797

41. Kołodziejczyk J, Ponczek MB. The role of fibrinogen, fibrin and fibrin(ogen) degradation products (FDPs) in tumor progression. Contemp Oncol (Pozn). 2013;17:113-119. doi:10.5114/ wo.2013.34611 
42. Nishida N, Yano H, Nishida T, Kamura T, Kojiro M. Angiogenesis in cancer. Vasc Health Risk Manag. 2006;2:213-219.

43. Garcia-Martinez R, Caraceni P, Bernardi M, Gines P, Arroyo V, Jalan R. Albumin: pathophysiologic basis of its role in the treatment of cirrhosis and its complications. Hepatology. 2013;58:1836-1846. doi: $10.1002 /$ hep. 26338

44. Gatta A, Verardo A, Bolognesi M, et al. Hypoalbuminemia. Intern Emerg Med. 2012;7:193-199. doi:10.1007/s11739-011-0732-2

45. Barbosa-Silva MC. Subjective and objective nutritional assessment methods: what do they really assess? Curr Opin Clin Nutr Metab Care. 2008;11:248-254. doi:10.1097/MCO.0b013e3282fba5d7

46. Seaton K. Albumin concentration controls cancer. J Natl Med Assoc. 2001;93:490-493.

47. Brenner DA, Buck M, Feitelberg SP, Chojkier M. Tumor necrosis factoralpha inhibits albumin gene expression in a murine model of cachexia. J Clin Invest. 1990;85:248-255. doi:10.1172/ JCI114419
48. Laursen I, Briand P, Lykkesfeldt AE. Serum albumin as a modulator on growth of the human breast cancer cell line, MCF-7. Anticancer Res. 1990;10:343-351.

49. Ouyang X, Dang Y, Zhang F, Huang Q. Low serum albumin correlates with poor survival in gastric cancer patients. Clin Lab. 2018;64:239-245. doi:10.7754/Clin.Lab.2017.170804

50. Huang J, Wang Y, Yuan Y, et al. Preoperative serum pre-albumin as an independent prognostic indicator in patients with localized upper tract urothelial carcinoma after radical nephroureterectomy. Oncotarget. 2017;8:36772-36779. doi:10.18632/oncotarget.13694

51. González-Trejo S, Carrillo JF, Carmona-Herrera DD, et al. Baseline serum albumin and other common clinical markers are prognostic factors in colorectal carcinoma: a retrospective cohort study. Medicine (Baltimore). 2017;96:e6610. doi:10.1097/MD.000000000 0006610 


\section{Supplementary materials}

Literature search strategy in PUBMED

(((albumin-to-fibrinogen[All Fields] OR albumin/fibrinogen[All Fields]) OR fibrinogen-to-albumin[All Fields])

OR fibrinogen/albumin[All Fields]) AND

(((("neoplasms"[MeSH Terms] OR “neoplasms"[All

Fields] OR “cancer"[All Fields]) OR ("tumour"[All

Fields] OR "neoplasms"[MeSH Terms] OR
"neoplasms"[All Fields] OR "tumor"[All Fields])) OR ("neoplasms"[MeSH Terms] OR "neoplasms"[All Fields] OR "malignancy"[All Fields])) OR ("carcinoma"[MeSH Terms] OR “carcinoma"[All Fields])) OR ("neoplasms"[MeSH Terms] OR "neoplasms"[All Fields])) AND ("loattrfull text"[sb] AND （"0001/01/01"[PDAT]: “2018/11/21"[PDAT]) AND English[lang]). 


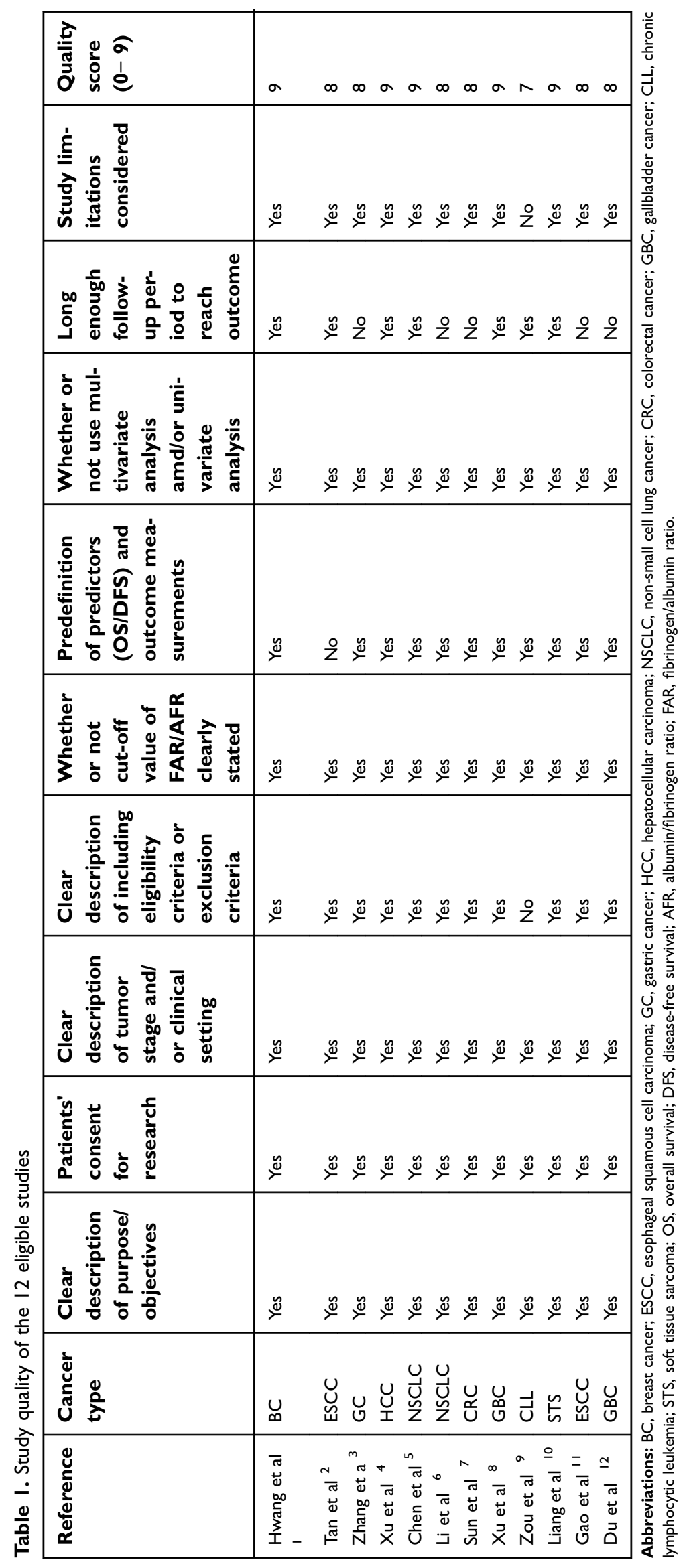




\section{References}

1. Hwang KT, Chung JK, Roh EY, et al. Prognostic influence of preoperative fibrinogen to albumin ratio for breast cancer. $J$ Breast Cancer. 2017;20:254-263. doi:10.4048/jbc.2017.20.3.254

2. Tan Z, Zhang M, Han Q, et al. A novel blood tool of cancer prognosis in esophageal squamous cell carcinoma: the fibrinogen/albumin ratio. J Cancer. 2017;8:1025-1029. doi:10.7150/jca.16491

3. Zhang J, Li SQ, Liao ZH, et al. Prognostic value of a novel FPR biomarker in patients with surgical stage II and III gastric cancer. Oncotarget. 2017;8:75195-75205. doi:10.18632/oncotarget.20661

4. Xu Q, Yan Y, Gu S, et al. A novel inflammation-based prognostic score: the fibrinogen/albumin ratio predicts prognoses of patients after curative resection for hepatocellular carcinoma. $J$ Immunol Res. 2018;2018:1-11. doi:10.1155/2018/4925498

5. Chen S, Yan H, Du J, et al. Prognostic significance of pre-resection albumin/fibrinogen ratio in patients with non-small cell lung cancer: a propensity score matching analysis. Clin Chim Acta. 2018;482:203208. doi:10.1016/j.cca.2018.04.012

6. Li SQ, Jiang YH, Lin J, et al. Albumin-to-fibrinogen ratio as a promising biomarker to predict clinical outcome of non-small cell lung cancer individuals. Cancer Med. 2018;7:1221-1231. doi:10.1002/cam4.1428
7. Sun F, Peng HX, Gao QF, et al. Preoperative circulating FPR and CCF score are promising biomarkers for predicting clinical outcome of stage II-III colorectal cancer patients. Cancer Manag Res. 2018;10:2151-2161. doi:10.2147/CMAR.S167398

8. Xu WY, Zhang HH, Xiong JP, et al. Prognostic significance of the fibrinogen-to-albumin ratio in gallbladder cancer patients. World J Gastroenterol. 2018;24:3281-3292. doi:10.3748/wjg. v24.i29.3281

9. Zou YX, Qiao J, Zhu HY, et al. Albumin-to-fibrinogen ratio as an independent prognostic parameter in untreated chronic lymphocytic leukemia: a retrospective study of 191 cases. Cancer Res Treat. 2018. doi:10.4143/crt.2018.358

10. Liang Y, Wang W, Que Y, et al. Prognostic value of the fibrinogen/ albumin ratio (FAR) in patients with operable soft tissue sarcoma. BMC Cancer. 2018;18:942. doi:10.1186/s12885-018-4242-8

11. Gao QF, Qiu JC, Huang XH, et al. The predictive and prognostic role of a novel ADS score in esophageal squamous cell carcinoma patients undergoing esophagectomy. Cancer Cell Int. 2018;18:153. doi:10.1186/s12935-018-0648-2

12. Du JH, Lu J. Circulating CEA-dNLR score predicts clinical outcome of metastatic gallbladder cancer patient. J Clin Lab Anal. 2019;33: e22684. doi:10.1002/jcla.22658
Cancer Management and Research

\section{Publish your work in this journal}

Cancer Management and Research is an international, peer-reviewed open access journal focusing on cancer research and the optimal use of preventative and integrated treatment interventions to achieve improved outcomes, enhanced survival and quality of life for the cancer patient.
The manuscript management system is completely online and includes a very quick and fair peer-review system, which is all easy to use. Visit http://www.dovepress.com/testimonials.php to read real quotes from published authors. 